\title{
Bifurcation Analysis of Gene Propagation Model Governed by Reaction-Diffusion Equations
}

\author{
Guichen Lu \\ School of Mathematics and Statistics, Chongqing University of Technology, Chongqing 400054, China \\ Correspondence should be addressed to Guichen Lu; bromn006@gmail.com
}

Received 3 April 2016; Revised 15 June 2016; Accepted 22 June 2016

Academic Editor: Andrew Pickering

Copyright (C) 2016 Guichen Lu. This is an open access article distributed under the Creative Commons Attribution License, which permits unrestricted use, distribution, and reproduction in any medium, provided the original work is properly cited.

We present a theoretical analysis of the attractor bifurcation for gene propagation model governed by reaction-diffusion equations. We investigate the dynamical transition problems of the model under the homogeneous boundary conditions. By using the dynamical transition theory, we give a complete characterization of the bifurcated objects in terms of the biological parameters of the problem.

\section{Introduction}

As the field of gene technology develops, the gene propagation problems continue to be relevant. Some recent advances and problems include the following: the genetic engineering for improving crop pest and disease resistance; the bacteria have developed a tolerance to widely prescribed antibiotics; the human genome project will enable us to deduce more information on human bodies and to deduce historical patterns of migration by archaeologists. Lots of papers developed equations to describe the changes in the frequency of alleles in a population that has several possible alleles at the locus in question. Fisher [1] proposed a reaction-diffusion equation with quadratic source term that models the spread of a recessive advantageous gene through a population that previously had only one allele at the locus in question. Fisher's equation is

$$
\frac{\partial u}{\partial t}=\theta \frac{\partial^{2} u}{\partial x^{2}}+r u(1-u),
$$

where $u$ is the frequency of the new mutant gene, $\theta$ is the diffusion coefficient, and $r$ is the intensity of selection in favor of the mutant gene.

In [2-5], the authors have claimed that a cubic source term was more appropriate than a quadratic source term. Although the cubic source term is implicit as one possibility in the general genetic dispersion equations derived by others, its significance has not been highlighted and the difference between cubic and quadratic source terms has not been examined. Based on the Fitzhugh-Nagumo equation and Huxley equation, by using the methods of a continuum limit of a discrete generation model, direct continuum modelling, and Fick's laws for random motion, Bradshaw-Hajek and Broadbridge [6-8] have derived a reaction-diffusion equation describing the spread of a new mutant gene; that is,

$$
\frac{\partial u}{\partial t}=\theta \frac{\partial^{2} u}{\partial x^{2}}+r u^{2}(1-u),
$$

where $u$ is the frequency of the new mutant gene, $\theta$ is the diffusion coefficient, and $r$ is the intensity of selection in favor of the mutant gene.

In [9-11], the authors have discussed the two possible alleles while some others recently investigate another case in which there are more than two possible alleles at the locus in question. For three possible alleles, Littler [12] has mostly used stochastic models while Bradshaw-Hajek and Broadbridge [6-8] have developed the reaction-diffusionconvection models.

In this paper, we will follow the work of Bradshaw-Hajek et al. [7] and investigate the gene propagation model of three possible alleles at the locus. By introducing the spatial two-dimensional domains, we will give a detailed analysis of the dynamical properties for the model and consider the attractor bifurcation to show a complete characterization of 
the attractors and their basins of attraction in terms of the physical parameters of the problem which is developed by $\mathrm{Ma}$ and Wang $[13,14]$.

The paper is organized as follows. In Section 2, we briefly summarize the two-dimensional spatial gene propagation model and give some mathematical settings. Section 3 states principle of exchange of stability for system. Section 4 is the main results of the phase transition theorems based on the attractor bifurcation theory. An example with the computer simulation of the pattern formation is given in the concluding remark section to illustrate our main results.

\section{Modelling Analysis}

In order to describe the spread of a new mutant gene, based on Skellam's method, Bradshaw-Hajek and Broadbridge [6] have developed a one-dimensional population genetics model governed by reaction-diffusion equation describing the changes in allelic frequencies. For a population having one new mutant allele $A_{1}$ and two original alleles $A_{2}, A_{3}$, there are six possible genotypes:

$$
A_{1} A_{1}, A_{1} A_{2}, A_{1} A_{3}, A_{2} A_{2}, A_{2} A_{3}, A_{3} A_{3} \text {. }
$$

Let $\rho_{i j}\left(x_{1}, x_{2}, t\right),(i, j=1,2,3)$ denote the frequency of individuals of the genotype $A_{i} A_{j}$ on the spatial two-dimensional domain. We follow the ideas of [6] and write the genotype equations as

$$
\begin{aligned}
& \frac{\partial \rho_{11}}{\partial t}=\Delta \rho_{11}-\mu \rho_{11}+\gamma_{11} u_{1}^{2} \rho \\
& \frac{\partial \rho_{12}}{\partial t}=\Delta \rho_{12}-\mu \rho_{12}+2 \gamma_{12} u_{1} u_{2} \rho, \\
& \frac{\partial \rho_{13}}{\partial t}=\Delta \rho_{13}-\mu \rho_{13}+2 \gamma_{13} u_{1}\left(1-u_{1}-u_{2}\right) \rho, \\
& \frac{\partial \rho_{22}}{\partial t}=\Delta \rho_{22}-\mu \rho_{22}+2 \gamma_{12} u_{2}^{2} \rho, \\
& \frac{\partial \rho_{23}}{\partial t}=\Delta \rho_{23}-\mu \rho_{23}+2 \gamma_{23} u_{2}\left(1-u_{1}-u_{2}\right) \rho, \\
& \frac{\partial \rho_{33}}{\partial t}=\Delta \rho_{33}-\mu \rho_{33}+2 \gamma_{33}\left(1-u_{1}-u_{2}\right)^{2} \rho,
\end{aligned}
$$

where $\Delta=\partial^{2} / \partial x_{1}{ }^{2}+\partial^{2} / \partial x_{2}{ }^{2}, \quad u_{i}\left(x_{1}, x_{2}, t\right)$ is the frequency of allele $A_{i}$, which can be expressed as

$$
\begin{aligned}
& u_{1}=\frac{2 \rho_{11}+\rho_{12}+\rho_{13}}{2 \rho}, \\
& u_{2}=\frac{\rho_{12}+2 \rho_{22}+\rho_{23}}{2 \rho}, \\
& u_{3}=1-u_{1}-u_{2},
\end{aligned}
$$

$\rho(x, t)$ is the total population density, $\mu$ is the common death rate, and $\gamma_{i j}$ is the reproductive success rate of individuals with genotype $A_{i} A_{j}$ for $i, j=1,2,3$, respectively.
From (5), we can simplify these above six equations into the following two coupled equations describing the change in frequency of two of alleles:

$$
\begin{gathered}
\frac{\partial u_{1}}{\partial t}=\Delta u_{1}+\frac{2}{\rho} \frac{\partial \rho}{\partial x} \frac{\partial u_{1}}{\partial x}+\Phi\left(u_{1}, u_{2}\right), \\
\frac{\partial u_{2}}{\partial t}=\Delta u_{2}+\frac{2}{\rho} \frac{\partial \rho}{\partial x} \frac{\partial u_{2}}{\partial x}+\Psi\left(u_{1}, u_{2}\right),
\end{gathered}
$$

where

$$
\begin{aligned}
\Phi\left(u_{1}, u_{2}\right)= & \left(\gamma_{13}-\gamma_{33}\right) u_{1}+\left(\gamma_{11}-3 \gamma_{13}+2 \gamma_{33}\right) u_{1}^{2} \\
& +\left(\gamma_{12}-\gamma_{13}-2 \gamma_{23}+2 \gamma_{33}\right) u_{1} u_{2} \\
& +\left(-\gamma_{22}+2 \gamma_{23}-\gamma_{33}\right) u_{1} u_{2}^{2} \\
& +\left(-2 \gamma_{12}+2 \gamma_{13}+2 \gamma_{23}-2 \gamma_{33}\right) u_{1}^{2} u_{2} \\
& +\left(-\gamma_{11}+2 \gamma_{13}-\gamma_{33}\right) u_{1}^{3} \\
\Psi\left(u_{1}, u_{2}\right)= & \left(\gamma_{23}-\gamma_{33}\right) u_{2}+\left(\gamma_{22}-3 \gamma_{23}+2 \gamma_{33}\right) u_{2}^{2} \\
& +\left(\gamma_{12}-2 \gamma_{13}-\gamma_{23}+2 \gamma_{33}\right) u_{1} u_{2} \\
& +\left(-\gamma_{11}+2 \gamma_{13}-\gamma_{33}\right) u_{1}^{2} u_{2} \\
& +\left(-2 \gamma_{12}+2 \gamma_{13}+2 \gamma_{23}-2 \gamma_{33}\right) u_{1} u_{2}^{2} \\
& +\left(-\gamma_{22}+2 \gamma_{23}-\gamma_{33}\right) u_{2}^{3} .
\end{aligned}
$$

Assume that the total population density is constant across the range (so that $\partial \rho / \partial x=0$ ); system (6) becomes

$$
\begin{aligned}
& \frac{\partial u_{1}}{\partial t}=\Delta u_{1}+\Phi\left(u_{1}, u_{2}\right), \\
& \frac{\partial u_{2}}{\partial t}=\Delta u_{2}+\Psi\left(u_{1}, u_{2}\right) .
\end{aligned}
$$

One of the attractions of (8) to mathematicians is to study the diffusion induced instability introduced by Turing in his 1952 seminal paper [15]. For showing the diffusion effect on stability, we will consider a modified equation of (8):

$$
\begin{aligned}
\frac{\partial u_{1}}{\partial t}= & \Delta u_{1}+\left(\gamma_{13}-\gamma_{33}\right) u_{1}+a_{1} u_{1}^{2}+a_{2} u_{1} u_{2}+a_{3} u_{1} u_{2}^{2} \\
& +a_{4} u_{1}^{2} u_{2}+a_{5} u_{1}^{3} \\
\frac{\partial u_{2}}{\partial t}= & d \Delta u_{2}+\left(\gamma_{23}-\gamma_{33}\right) u_{2}+b_{1} u_{2}^{2}+b_{2} u_{1} u_{2}+a_{4} u_{1} u_{2}^{2} \\
& +a_{5} u_{1}^{2} u_{2}+a_{3} u_{2}^{3}
\end{aligned}
$$

where $a_{1}=\gamma_{11}-3 \gamma_{13}+2 \gamma_{33}, a_{2}=\gamma_{12}-\gamma_{13}-2 \gamma_{23}+2 \gamma_{33}$, $a_{3}=-\gamma_{22}+2 \gamma_{23}-\gamma_{33}, a_{4}=-2 \gamma_{12}+2 \gamma_{13}+2 \gamma_{23}-2 \gamma_{33}$, $a_{5}=-\gamma_{11}+2 \gamma_{13}-\gamma_{33}, b_{1}=\gamma_{22}-3 \gamma_{23}+2 \gamma_{33}, b_{2}=\gamma_{12}-2 \gamma_{13}-$ $\gamma_{23}+2 \gamma_{33}$, and $a_{3}^{2}+a_{4}^{2}+a_{5}^{2} \neq 0 . d$ is the diffusion coefficient which measures the dispersal rate of allele $A_{2}$. On the other hand, diffusive terms can be considered as describing 
the ability of the allele $A_{i}$ to occupy different zones in 2dimensional space either through the action of small-scale mechanism or by some native transport device.

System (9) has seven constant solutions: $\left(u_{1}, u_{2}\right)=$ $(0,0),(0,1),(1,0),(0, \alpha),(\beta, 0),\left(a^{*}, b^{*}\right),(a, b)$, where $\alpha, \beta, a^{*}$, $b^{*}, a$, and $b$ are complicated expressions of the reproductive success rate $\gamma_{i j}$ and $a, b$ are given by

$$
\begin{aligned}
& a=\frac{\Delta_{1}}{\Delta_{0}}, \\
& b=\frac{\Delta_{2}}{\Delta_{0}},
\end{aligned}
$$

where

$$
\begin{aligned}
\Delta_{1}= & -\gamma_{13} \gamma_{23}+\gamma_{13} \gamma_{22}-\gamma_{12} \gamma_{23}+\gamma_{12} \gamma_{33}-\gamma_{22} \gamma_{33} \\
& +\gamma_{23}{ }^{2}, \\
\Delta_{2}= & -\gamma_{12} \gamma_{13}+\gamma_{12} \gamma_{33}-\gamma_{13} \gamma_{23}-\gamma_{11} \gamma_{33}+\gamma_{11} \gamma_{23} \\
& +\gamma_{13}{ }^{2}, \\
\Delta_{0}= & \gamma_{12}{ }^{2}+\gamma_{13}{ }^{2}+\gamma_{23}{ }^{2}-2 \gamma_{12} \gamma_{13}-2 \gamma_{12} \gamma_{23}-\gamma_{11} \gamma_{33} \\
& +2 \gamma_{12} \gamma_{33}-\gamma_{11} \gamma_{22}-2 \gamma_{13} \gamma_{23}-\gamma_{22} \gamma_{33} \\
& +2 \gamma_{13} \gamma_{22}+2 \gamma_{11} \gamma_{23} .
\end{aligned}
$$

Considering the biological context, we assume that the steady state solution $(a, b)$ is positive and $0<u_{1}, u_{2}<1$.

In the present paper, we focus on the bifurcation from the constant solution $(a, b)$. Let

$$
\begin{aligned}
& u_{1}=a+u_{1}^{\prime}, \\
& u_{2}=b+u_{2}^{\prime} .
\end{aligned}
$$

Omitting the primes, then system (9) becomes

$$
\begin{aligned}
\frac{\partial u_{1}}{\partial t}= & \Delta u_{1}+a\left(\gamma_{11}-\gamma_{13}\right) u_{1}+a\left(\gamma_{12}-\gamma_{13}\right) u_{2} \\
& +G_{1}\left(u_{1}, u_{2}\right)
\end{aligned}
$$

$$
\begin{aligned}
& H=L^{2}\left(\Omega, R^{2}\right) \\
& H_{1}= \begin{cases}\left\{H^{2}\left(\Omega, R^{2}\right) \cap H_{0}^{1}\left(\Omega, R^{2}\right)\right\} & \text { for boundary conditions (15) } \\
\left\{u \in H^{2}\left(\Omega, R^{2}\right)\left|\frac{\partial u}{\partial n}\right|_{\partial \Omega}=0\right\} & \text { for boundary conditions (16). }\end{cases}
\end{aligned}
$$

It is clear that $H$ and $H_{1}$ are two Hilbert space and $H_{1} \hookrightarrow H$ is dense and compact inclusion.

Later, we choose the bifurcation parameter $\lambda$ to be the diffusion coefficient $d$; that is, $\lambda=d$. Let $L_{\lambda}: H_{1} \rightarrow H$ be defined by

$$
\begin{aligned}
\frac{\partial u_{2}}{\partial t}= & d \Delta u_{2}+b\left(\gamma_{12}-\gamma_{23}\right) u_{1}+b\left(\gamma_{22}-\gamma_{23}\right) u_{2} \\
& +G_{2}\left(u_{1}, u_{2}\right)
\end{aligned}
$$

where

$$
\begin{aligned}
G_{1}\left(u_{1}, u_{2}\right)= & \left(a_{4} b+3 a_{5} a+a_{1}\right) u_{1}{ }^{2} \\
& +\left(a_{2}+2 a_{3} b+2 a_{4} a\right) u_{1} u_{2}+a a_{3} u_{2}{ }^{2} \\
& +a_{5} u_{1}{ }^{3}+u_{1} a_{3} u_{2}{ }^{2}+a_{4} u_{1}{ }^{2} u_{2}, \\
G_{2}\left(u_{1}, u_{2}\right)= & b a_{5} u_{1}^{2}+\left(2 a_{5} a+b_{2}+2 a_{4} b\right) u_{1} u_{2} \\
& +\left(b_{1}+3 a_{3} b+a_{4} a\right) u_{2}{ }^{2}+a_{3} u_{2}{ }^{3} \\
& +a_{4} u_{1} u_{2}{ }^{2}+a_{5} u_{2} u_{1}{ }^{2} .
\end{aligned}
$$

We assume that system (13) is satisfied on an open bounded domain $\Omega \subset R^{2}$. There are two types of biologically sound boundary conditions: the Dirichlet boundary condition,

$$
\begin{aligned}
& \left.u_{1}\right|_{\partial \Omega}=0, \\
& \left.u_{2}\right|_{\partial \Omega}=0,
\end{aligned}
$$

which means that the frequency is extinct in the boundary of range and the Neumann boundary condition:

$$
\begin{aligned}
& \left.\frac{\partial u_{1}}{\partial n}\right|_{\partial \Omega}=0 \\
& \left.\frac{\partial u_{2}}{\partial n}\right|_{\partial \Omega}=0
\end{aligned}
$$

which means that the frequency is invariant in the boundary of range in biological significant.

Define the function spaces

where

$$
\begin{aligned}
-A_{\lambda} u & =\left(\Delta u_{1}, \lambda \Delta u_{2}\right)^{T}, \\
B u & =\left(\begin{array}{ll}
a_{11} & a_{12} \\
a_{21} & a_{22}
\end{array}\right)\left(\begin{array}{l}
u_{1} \\
u_{2}
\end{array}\right),
\end{aligned}
$$

where $u=\left(u_{1}, u_{2}\right)^{T} \in H_{1}, a_{11}=a\left(\gamma_{11}-\gamma_{13}\right), a_{12}=a\left(\gamma_{12}-\right.$ $\left.\gamma_{13}\right), a_{21}=b\left(\gamma_{12}-\gamma_{23}\right)$, and $a_{22}=b\left(\gamma_{22}-\gamma_{23}\right)$. 
Furthermore, let $G\left(u_{1}, u_{2}\right)$ be given by

$$
\begin{aligned}
& G\left(u_{1}, u_{2}\right)=\left(G_{1}^{2}\left(u_{1}, u_{2}\right)+G_{1}^{3}\left(u_{1}, u_{2}\right), G_{2}^{2}\left(u_{1}, u_{2}\right)\right. \\
& \left.\quad+G_{2}^{3}\left(u_{1}, u_{2}\right)\right)^{T},
\end{aligned}
$$

where

$$
\begin{aligned}
& \left(\begin{array}{l}
G_{2}^{1}\left(u_{1}, u_{2}\right) \\
G_{2}^{2}\left(u_{1}, u_{2}\right)
\end{array}\right) \\
& =\left(\begin{array}{l}
\left(a_{4} b+3 a_{5} a+a_{1}\right) u_{1}^{2}+\left(a_{2}+2 a_{3} b+2 a_{4} a\right) u_{1} u_{2}+a a_{3} u_{2}^{2} \\
b a_{5} u_{1}^{2}+\left(2 a_{5} a+b_{2}+2 a_{4} b\right) u_{1} u_{2}+\left(b_{1}+3 a_{3} b+a_{4} a\right) u_{2}^{2}
\end{array}\right), \\
& \left(\begin{array}{l}
G_{3}^{1}\left(u_{1}, u_{2}\right) \\
G_{3}^{2}\left(u_{1}, u_{2}\right)
\end{array}\right)=\left(\begin{array}{l}
a_{5} u_{1}^{3}+u_{1} a_{3} u_{2}^{2}+a_{4} u_{1}^{2} u_{2} \\
a_{3} u_{2}^{3}+a_{4} u_{1} u_{2}^{2}+a_{5} u_{1}^{2} u_{2}
\end{array}\right) .
\end{aligned}
$$

Then (13) can be written in the following operator form:

$$
\frac{d u}{d t}=L_{\lambda} u+G(u)
$$

\section{Principle of Exchange of Stability}

From the theoretical ecology, it is interesting to study the bifurcation of system (9) at steady state $(a, b)$. Bifurcation means that a change in the stability or in the types of steady state which occurs as a parameter is varied in a dissipative dynamic system; that is, the state changes during the biology conditions. The classical bifurcation types are Hopf bifurcation and Turing bifurcation. Ma and Wang $[13,14]$ have developed new methods to study bifurcations and transitions which are called attractor bifurcations. This theory yields complete information about bifurcations, transitions, stability, and persistence, including information about transient states, in terms of the physical parameters of the system. Therefore, in this section, we consider the attractor bifurcation of system $(9)$ at $(a, b)$, and from the transformation, we only need to discuss system $(13)$ at $(0,0)$.

Firstly, we consider the linear system of (13),

$$
\begin{aligned}
& \frac{\partial u_{1}}{\partial t}=\Delta u_{1}+a_{11} u_{1}+a_{12} u_{2}, \\
& \frac{\partial u_{2}}{\partial t}=\lambda \Delta u_{2}+a_{21} u_{1}+a_{22} u_{2}
\end{aligned}
$$

and its eigenvalue problem

$$
\begin{aligned}
L_{\lambda} \varphi & =\beta(\lambda) \varphi, \quad \varphi \in H_{1}, \\
-\Delta e_{k} & =\rho_{k} e_{k}
\end{aligned}
$$

with the Dirichlet boundary condition (15) or the Neumann boundary condition (16).

Let $M_{k}$ be defined by

$$
M_{k}=\left(\begin{array}{cc}
a_{11}-\rho_{k} & a_{12} \\
a_{21} & a_{22}-\lambda \rho_{k}
\end{array}\right) .
$$

It is easy to see that any eigenvector $\phi_{k}$ and eigenvalue $\beta_{k}$ of (24) can be expressed as

$$
\begin{array}{r}
\phi_{k}=\left(\begin{array}{c}
\xi_{k 1} e_{k} \\
\xi_{k 2} e_{k}
\end{array}\right), \\
M_{k}^{\lambda}\left(\begin{array}{c}
\xi_{k 1} \\
\xi_{k 2}
\end{array}\right)=\beta_{k}\left(\begin{array}{l}
\xi_{k 1} \\
\xi_{k 2}
\end{array}\right),
\end{array}
$$

where $e_{k}$ is as in (25) and $\beta_{k}$ is also the eigenvalue of $B_{k}^{\lambda}$. By (24), $\beta_{k}$ can be written as

$$
\begin{aligned}
\beta_{1 k}= & -\frac{1}{2}\left[\left(\rho_{k}-a_{11}\right)+\left(\lambda \rho_{k}-a_{22}\right)\right] \\
& +\frac{1}{2} \sqrt{\left[\left(\rho_{k}-a_{11}\right)-\left(\lambda \rho_{k}-a_{22}\right)\right]^{2}+4 a_{12} a_{21}}, \\
\beta_{2 k}=- & \frac{1}{2}\left[\left(\rho_{k}-a_{11}\right)+\left(\lambda \rho_{k}-a_{22}\right)\right] \\
& -\frac{1}{2} \sqrt{\left[\left(\rho_{k}-a_{11}\right)-\left(\lambda \rho_{k}-a_{22}\right)\right]^{2}+4 a_{12} a_{21}} .
\end{aligned}
$$

It is clear that $\beta_{2 k}(\lambda)<\beta_{1 k}(\lambda)=0$ if and only if

$$
\begin{aligned}
\left(\rho_{k}-a_{11}\right)+\left(\lambda \rho_{k}-a_{22}\right) & >0, \\
\left(\rho_{k}-a_{11}\right)\left(\lambda \rho_{k}-a_{22}\right)-a_{12} a_{21} & =0 .
\end{aligned}
$$

We define a parameter

$$
\lambda_{0}=\frac{a_{22} \rho_{K}-\left(a_{11} a_{22}-a_{12} a_{21}\right)}{\left(\rho_{K}-a_{11}\right) \rho_{K}},
$$

where $\rho_{k}=\rho_{K}$ such that $\chi_{K}(\lambda)$ attains its minimum values:

$$
\begin{aligned}
\chi_{K}(\lambda) & =\min _{\rho_{k}}\left\{\lambda \rho_{k}+\frac{a_{11} a_{22}-a_{12} a_{21}}{\rho_{k}}-\left(\lambda a_{11}+a_{22}\right)\right\} \\
& =\lambda \rho_{K}+\frac{a_{11} a_{22}-a_{12} a_{21}}{\rho_{K}}-\left(\lambda a_{11}+a_{22}\right) .
\end{aligned}
$$

Theorem 1. Let $\lambda_{0}$ be the number given in (30) such that (29) is satisfied and let $K \geq 1$ be the integer such that the minimum is achieved at $\rho_{K}$. Then $\beta_{1 K}(\lambda)$ is the first real eigenvalue of $L_{\lambda}$ near $\lambda=\lambda_{0}$ satisfying that

$$
\begin{array}{cc}
\beta_{1 K}(\lambda) \begin{cases}<0, & \lambda \in \Lambda_{K}^{+}, \\
=0, & \lambda=\lambda_{0}, \\
>0, & \lambda \in \Lambda_{K}^{-},\end{cases} \\
\operatorname{Re} \beta_{2 k}\left(\lambda_{0}\right)<0, & \forall k \in \mathbb{N}, \\
\operatorname{Re} \beta_{1 k}\left(\lambda_{0}\right)<0, & \forall k \neq K .
\end{array}
$$

Here $\Lambda_{K}^{+}=\left\{\lambda \mid \chi_{K}(\lambda)>0\right\}$ and $\Lambda_{K}^{-}=\left\{\lambda \mid \chi_{K}(\lambda)<0\right\}$.

In the absence of diffusion, system (23) becomes the spatial homogeneous system

$$
\begin{aligned}
& \frac{d u_{1}}{d t}=a_{11} u_{1}+a_{12} u_{2}, \\
& \frac{d u_{2}}{d t}=a_{21} u_{1}+a_{22} u_{2} .
\end{aligned}
$$


System (33) is local asymptotic stability if

$$
\begin{array}{r}
a_{11}+a_{22}<0, \\
a_{11} a_{22}-a_{12} a_{21}>0,
\end{array}
$$

and the Hopf bifurcation occurs when

$$
\begin{aligned}
a_{11}+a_{22} & =0 \\
a_{11} a_{22}-a_{12} a_{21} & <0 .
\end{aligned}
$$

From Theorem 1, we can infer that if condition (34) and $\lambda \in \Lambda_{K}^{-}$hold, then the homogeneous attracting equilibrium loses stability due to the interaction of diffusion processes and system (23) undergoes a Turing bifurcation.

\section{Phase Transition on Homogeneous State}

Hereafter, we always assume that the eigenvalue $\beta_{1 K}(\lambda)$ in (24) is simple. Based on Theorem 1 , as $\lambda \in \Lambda_{K}^{-}$the transition of (22) occurs at $\lambda=\lambda_{0}$, which is from real eigenvalues.

The following is the main theorem in this paper, which provides not only a precise criterion for the transition types of (22) but also globally dynamical behaviors.

Theorem 2. Let $\rho_{K}$ be defined in Theorem 1 , and $e_{K}$ is the corresponding eigenvector to $\rho_{K}$ of (25) satisfying

$$
\int_{\Omega} e_{K}^{3} d x \neq 0
$$

For system (22), we have the following assertions.

(1) Equation (22) has a mixed transition from $\left(0, \lambda_{0}\right)$; more precisely, there exists a neighborhood $U \in X$ of $u=0$, such that $U$ is separated into two disjoint open sets $U_{1}^{\lambda}$ and $U_{2}^{\lambda}$ by the stable manifold $\Gamma_{\lambda}$ of $u=0$, satisfying the following.

(a) $U=U_{1}^{\lambda}+U_{2}^{\lambda}+\Gamma_{\lambda}$. (b) The transition in $U_{1}^{\lambda}$ is jump. (c) The transition in $U_{2}^{\lambda}$ is continuous.

(2) Equation (22) bifurcates in $U_{2}^{\lambda}$ to a unique singular point $v^{\lambda}$ on $\lambda \in \Lambda_{K}^{+}$which is attractor such that, for any $\phi \in U_{2}^{\lambda}$,

$$
\lim _{t \rightarrow \infty}\left\|u(t, \phi)-v^{\lambda}\right\|_{X}=0
$$

(3) Equation (22) bifurcates on $\lambda \in \Lambda_{K}^{-}$to a unique saddle point with morse index 1.

(4) The bifurcated singular point $v^{\lambda}$ can be expressed by

$$
v^{\lambda}=-\frac{\beta_{1 K}(\lambda)}{b} \varphi_{K}+o\left(\left\|\beta_{1 K}(\lambda)\right\|\right)
$$

Here

$$
\begin{aligned}
b= & \frac{1}{a_{12} a_{21}+\left(a_{11}-\rho_{K}\right)^{2}}\left[-b\left(b_{2}+a_{4} b+2 a_{5} a\right)\right. \\
& \cdot\left(a a_{3}\left(a\left(a_{1}+a_{4} b+2 a_{5} a\right)-\rho_{K}\right)^{2}\right. \\
& -\left(a_{2}+2 a_{3} b+2 a_{4} a\right) a\left(a_{2}+2 a_{3} b+a_{4} a\right) \\
& \cdot\left(a\left(a_{1}+a_{4} b+2 a_{5} a\right)-\rho_{K}\right)+\left(a_{4} b+3 a_{5} a+a_{1}\right) \\
& \left.\cdot a^{2}\left(a_{2}+2 a_{3} b+a_{4} a\right)^{2}\right)+\left(a\left(a_{1}+a_{4} b+2 a_{5} a\right)\right. \\
& \left.-\rho_{K}\right)\left(\left(b_{1}+3 a_{3} b+a_{4} a\right)\right. \\
& \cdot\left(a\left(a_{1}+a_{4} b+2 a_{5} a\right)-\rho_{K}\right)^{2}-\left(2 a_{5} a+b_{2}+2 a_{4} b\right) \\
& \cdot a\left(a_{2}+2 a_{3} b+a_{4} a\right)\left(a\left(a_{1}+a_{4} b+2 a_{5} a\right)-\rho_{K}\right) \\
& \left.\left.+b a_{5} a^{2}\left(a_{2}+2 a_{3} b+a_{4} a\right)^{2}\right)\right] .
\end{aligned}
$$

Proof. First, we need to get the reduced equation of (22) near $\lambda=\lambda_{0}$.

Let $u=x \cdot \phi_{K}+y$, where $y=\sum_{i=1}^{n} y_{i} \phi_{i}$ and $\phi_{K}$ is the eigenvector of (24) corresponding to $\beta_{1 K}(\lambda)$ at $\lambda=\lambda_{0}$. Then the reduced equation of $(22)$ reads

$$
\frac{d x}{d \mathrm{t}}=\beta_{1 K}\left(\lambda_{0}\right) x+\frac{1}{\left\langle\phi_{K}, \phi_{K}^{*}\right\rangle}\left\langle G\left(x \cdot \phi_{K}+y\right), \phi_{K}^{*}\right\rangle,
$$

Here $\phi_{K}^{*}$ is the conjugate eigenvector of $\phi_{K}$.

By Implicit Function Theorem, we can obtain that

$$
y=\Phi(x, \lambda)=o(\|x\|)
$$

Substituting (41) into (40), we get the bifurcation equation of (22) as follows:

$$
\begin{aligned}
\frac{d x}{d t}= & \beta_{1 K}\left(\lambda_{0}\right) x+\frac{1}{\left\langle\phi_{K}, \phi_{K}^{*}\right\rangle}\left\langle G\left(x \cdot \phi_{K}\right), \phi_{K}^{*}\right\rangle \\
& +o(2) .
\end{aligned}
$$

By $(27), \phi_{K}$ is written as

$$
\phi_{K}=\left(\xi_{1} e_{K}, \xi_{2} e_{K}\right)^{T}
$$

with $\left(\xi_{1}, \xi_{2}\right)$ satisfying

$$
\left(\begin{array}{cc}
a_{11}-\rho_{K} & a_{12} \\
a_{21} & a_{22}-\lambda \rho_{\mathrm{K}}
\end{array}\right)\left(\begin{array}{l}
\xi_{1} \\
\xi_{2}
\end{array}\right)=\beta_{1 K}\left(\lambda_{0}\right)\left(\begin{array}{l}
\xi_{1} \\
\xi_{2}
\end{array}\right),
$$

from which we get

$$
\left(\xi_{1}, \xi_{2}\right)=\left(-a_{12}, a_{11}-\rho_{K}\right)
$$

Likewise, $\phi_{K}^{*}$ is

$$
\phi_{K}^{*}=\left(\xi_{1}^{*} e_{K}, \xi_{2}^{*} e_{K}\right)^{T},
$$


with $\left(\xi_{1}^{*}, \xi_{2}^{*}\right)$ satisfying

$$
\left(\begin{array}{cc}
a_{11}-\rho_{K} & a_{21} \\
a_{12} & a_{22}-\lambda \rho_{K}
\end{array}\right)\left(\begin{array}{l}
\xi_{1} \\
\xi_{2}
\end{array}\right)=\beta_{1 K}\left(\lambda_{0}\right)\left(\begin{array}{l}
\xi_{1}^{*} \\
\xi_{2}^{*}
\end{array}\right),
$$

which yields

$$
\left(\xi_{1}^{*}, \xi_{2}^{*}\right)=\left(-a_{21}, a_{11}-\rho_{K}\right) .
$$

By (22), the nonlinear operator $G$ is

$$
\begin{aligned}
& \left(\begin{array}{l}
G_{2}^{1}\left(u_{1}, u_{2}\right) \\
G_{2}^{2}\left(u_{1}, u_{2}\right)
\end{array}\right) \\
& =\left(\begin{array}{l}
\left(a_{4} b+3 a_{5} a+a_{1}\right) u_{1}^{2}+\left(a_{2}+2 a_{3} b+2 a_{4} a\right) u_{1} u_{2}+a a_{3} u_{2}^{2} \\
b a_{5} u_{1}^{2}+\left(2 a_{5} a+b_{2}+2 a_{4} b\right) u_{1} u_{2}+\left(b_{1}+3 a_{3} b+a_{4} a\right) u_{2}^{2}
\end{array}\right) .
\end{aligned}
$$

Then, in view of (45) and (48), by direct computation we derive that

$$
\begin{aligned}
& \left\langle G\left(x \xi_{1} e_{K}, x \xi_{2} e_{K}\right), \phi_{K}^{*}\right\rangle=\left[\left(a_{4} b+a_{1}+3 a_{5} a\right) \xi_{1}{ }^{2} \xi_{1}^{*}\right. \\
& \quad+\xi_{2}^{*} a_{5} b \xi_{1}^{2}+\left(b_{2}+2 a_{5} a+2 a_{4} b\right) \xi_{2} \xi_{1} \xi_{2}^{*} \\
& +\left(b_{1}+a_{4} a+3 a_{3} b\right) \xi_{2}{ }^{2} \xi_{2}^{*} \\
& \left.+\left(2 a_{3} b+a_{2}+2 a_{4} a\right) \xi_{2} \xi_{1} \xi_{1}^{*}+\xi_{1}^{*} a_{3} a \xi_{2}^{2}\right] \\
& +x^{2} \int_{\Omega} e_{K}^{3} d x+o\left(x^{2}\right) .
\end{aligned}
$$

By (45) and (48), we have

$$
\left\langle\phi, \phi^{*}\right\rangle=\left[a_{12} a_{21}+\left(a_{11}-\rho_{K}\right)^{2}\right] \int_{\Omega} e_{K}^{2} d x
$$

Hence, by (50) the reduced equation (13) is expressed as

$$
\frac{d x}{d t}=\beta_{1 K}(\lambda) x+b x^{2}+o\left(x^{2}\right)
$$

where $b$ is the parameter as in (39). Based on Theorem A.2 in [16], this theorem follows from (52). The proof is complete.

Remark 3. If the domain $\Omega \neq(0, L) \times D$ with $D \in R$ being a bounded open set, then condition (36) holds true for boundary conditions (15) and (16).

If the domain $\Omega=(0, L) \times D$ with $D \in R$ being a bounded open set, then

$$
\int_{\Omega} e_{K}^{3} d x=0
$$

holds true for Neumann condition (16) and Dirichlet condition (15) when the number $m$ in $\rho_{k_{0}}=m^{2} \pi^{2} / L^{2}+\rho_{k_{0}}^{\prime}$ is even.

Hereafter, we consider the Neumann condition (16) and let the domain $\Omega=\left(0, L_{1}\right) \times\left(0, L_{2}\right)$ with $L_{1} \neq L_{2}$.

The following eigenvalue problem (24) with the equation

$$
\begin{aligned}
-\Delta e_{k} & =\rho_{k} e_{k}, \\
\left.\frac{\partial e_{k}}{\partial n}\right|_{\partial \Omega} & =0
\end{aligned}
$$

has eigenfunctions $e_{k}$ and eigenvalues $\rho_{k}$ as follows:

$$
\begin{aligned}
& e_{k}=\cos \frac{k_{1} \pi x_{1}}{L_{1}} \cos \frac{k_{2} \pi x_{2}}{L_{2}}, \\
& \quad k=\left(k_{1}, k_{2}\right), k_{1}, k_{2}=0,1,2, \ldots, \\
& \rho_{k}=\pi^{2}\left(\frac{k_{1}^{2}}{L_{1}^{2}}+\frac{k_{2}^{2}}{L_{2}^{2}}\right) .
\end{aligned}
$$

Here $\rho_{0}=0$ and $e_{0}=1$.

Denote $K=\left(K_{1}, K_{2}\right)$, the pair of integers satisfying (30), and simply denote $K_{1}=\left(K_{1}, 0\right)$ and $K_{2}=\left(0, K_{2}\right)$; then we have the following.

Theorem 4. Let $\eta$ be the parameter defined by

$$
\eta=\frac{\left[(9 / 16) \widetilde{H}\left(\xi, \xi^{*}\right)+(1 / 4) H\left(\xi, \xi^{*}\right)\right]}{a_{12} a_{21}+\left(a_{11}-\rho_{K}\right)^{2}},
$$

where $\widetilde{H}\left(\xi, \xi^{*}\right)=a_{3} \xi_{1}^{*} \xi_{1} \xi_{2}{ }^{2}+a_{4} \eta_{1} \xi_{1}{ }^{2} \xi_{2}+a_{5} \xi_{1}^{*} \xi_{1}{ }^{3}+b_{3} \eta_{2} \xi_{1}{ }^{2} \xi_{2}+$ $b_{4} \xi_{2}^{*} \xi_{1} \xi_{2},{ }^{2}+b_{5} \xi_{2}^{*} \xi_{2}{ }^{3}$ and $H\left(\xi, \xi^{*}\right)$ is in Appendix and $\xi, \xi^{*}$ are defined in the proof.

Assume that the eigenvalue $\beta_{1 K}(\lambda)$ satisfying (28) is simple; then for system (22), we have the following assertions:

(1) This system has a transition from $\left(0, \lambda_{0}\right)$; that is, $u=0$ is asymptotically stable for $\lambda \in \Lambda_{K}^{-}$and unstable for $\lambda \in \Lambda_{K}^{+}$which transits to a stable equilibrium at $\lambda=$ $\lambda_{0}$.

(2) If $\eta>0$, then (22) has a jump transition from $\left(0, \lambda_{0}\right)$ and bifurcates on $\lambda \in \Lambda_{K}^{+}$to exactly two saddle points $v_{1}^{\lambda}$ and $v_{2}^{\lambda}$ with the Morse index one.

(3) If $\eta<0$, then (22) has a continuous transition from $\left(0, \lambda_{0}\right)$, which is an attractor bifurcation.

(4) The bifurcated singular points $v_{1}^{\lambda}$ and $v_{2}^{\lambda}$ in the above cases can be expressed in the following form:

$$
v_{1,2}^{\lambda}= \pm\left|\frac{\beta_{1 K}(\lambda)}{\eta}\right|^{1 / 2} e_{K}+o\left(\left\|\beta_{1 K}\right\|^{1 / 2}\right) .
$$

Proof. Assertion (1) follows from (32). To prove assertions (2) and (3), we need to get the reduced equation of (22) to the center manifold near $\lambda=\lambda_{0}$.

Let $u=x \cdot \varphi_{K}+\Phi$, where $\varphi_{K}$ is the eigenvector of (25) corresponding to $\beta_{1 K}(\lambda)$ at $\lambda=\lambda_{0}$ and $\Phi(x)$ the center manifold function of (22). Then the reduced equation of (22) takes the following form:

$$
\frac{d x}{d t}=\beta_{1 K}\left(\lambda_{0}\right) x+\frac{1}{\left\langle\varphi_{K}, \varphi_{K}^{*}\right\rangle}\left\langle G\left(x \cdot \varphi_{K}+\Phi\right), \varphi_{K}^{*}\right\rangle .
$$

Here $\varphi_{K}^{*}$ is the conjugate eigenvector of $\varphi_{K}$.

By $(27), \varphi_{K}$ is written as

$$
\varphi_{K}=\left(\xi_{1} e_{K}, \xi_{2} e_{K}\right)^{T},
$$

with $\left(\xi_{1}, \xi_{2}\right)$ satisfying (45). 
Likewise, $\varphi_{K}^{*}$ is

$$
\varphi_{K}^{*}=\left(\xi_{1}^{*} e_{K}, \xi_{2}^{*} e_{K}\right)^{T},
$$

with $\left(\xi_{1}^{*}, \xi_{2}^{*}\right)$ satisfying $(46)$.

It is known that the center manifold function

$$
\Phi(x)=\left(\Phi_{1}(x), \Phi_{2}(x)\right)=o\left(x^{2}\right) .
$$

By using the formula for center manifold functions in [13, $14,16], \Phi=\left(\Phi_{1}, \Phi_{2}\right)$ satisfies

$$
\begin{aligned}
L_{\lambda_{0}} \Phi & =-x^{2} G_{2}\left(\xi_{1} e_{k}, \xi_{2} e_{K}\right)+o\left(x^{2}\right) \\
& =-x^{2} e_{K}^{2}\left(\begin{array}{l}
\eta_{1} \\
\eta_{2}
\end{array}\right)+o\left(x^{2}\right)
\end{aligned}
$$

where

$$
\begin{aligned}
\eta_{1}= & a a_{3} \xi_{2}^{2}+\left(a_{2}+2 a_{4} a+2 a_{3} b\right) \xi_{1} \xi_{2} \\
& +\left(a_{4} b+3 a_{5} a+a_{1}\right) \xi_{1}{ }^{2}, \\
\eta_{2}= & \left(b_{1}+3 a_{3} b+a_{4} a\right) \xi_{2}{ }^{2}+\left(2 a_{5} a+2 a_{4} b+b_{2}\right) \xi_{1} \xi_{2} \\
& +b a_{5} \xi_{1}{ }^{2} .
\end{aligned}
$$

From (55), we see that

$$
\begin{aligned}
e_{K}^{2} & =\frac{1}{4}\left(1+e_{2 K_{1}}\right)\left(1+e_{2 K_{2}}\right) \\
& =\frac{1}{4}\left(e_{0}+e_{2 K_{1}}+e_{2 K_{2}}+e_{2 K}\right) .
\end{aligned}
$$

Let

$$
\begin{aligned}
\left(\begin{array}{c}
\Phi_{1} \\
\Phi_{2}
\end{array}\right)= & \left(\begin{array}{c}
\Phi_{1}^{0} \\
\Phi_{2}^{0}
\end{array}\right) e_{0}+\left(\begin{array}{c}
\Phi_{1}^{2 K_{1}} \\
\Phi_{2}^{2 K_{1}}
\end{array}\right) e_{2 K_{1}}+\left(\begin{array}{c}
\Phi_{1}^{2 K_{2}} \\
\Phi_{2}^{2 K_{2}}
\end{array}\right) e_{2 K_{2}} \\
& +\left(\begin{array}{c}
\Phi_{1}^{2 K} \\
\Phi_{2}^{2 K}
\end{array}\right) e_{2 K} .
\end{aligned}
$$

It is clear that

$$
L_{\lambda_{0}}\left(\begin{array}{c}
\Phi_{1}^{k} \\
\Phi_{2}^{k}
\end{array}\right) e_{k}=M_{k}\left(\begin{array}{c}
\Phi_{1}^{k} \\
\Phi_{2}^{k}
\end{array}\right) e_{k}
$$

where $M_{k}$ is the matrix given by (26). Then, it follows from (65) and (66) that

$$
\left(\begin{array}{l}
\Phi_{1}^{k} \\
\Phi_{2}^{k}
\end{array}\right)=-\frac{1}{4} M_{k}^{-1}\left(\begin{array}{l}
\eta_{1} \\
\eta_{2}
\end{array}\right)+o\left(x^{2}\right)
$$

for $k=K, K_{1}, K_{2}$, where

$$
\begin{aligned}
M_{k}^{-1}= & \frac{1}{\operatorname{det}\left(M_{k}\right)}\left[\begin{array}{cc}
-\lambda_{0} \rho_{k}+a_{22} & -a_{12} \\
-a_{21} & -\rho_{k}+a_{11}
\end{array}\right], \\
\operatorname{det}\left(M_{k}\right)= & \lambda_{0} \rho_{k}^{2}-\rho_{k} a_{22}-a_{11} \lambda_{0} \rho_{k}+a_{11} a_{22} \\
& -a_{12} a_{21} .
\end{aligned}
$$

Direct calculation shows that

$$
\left(\begin{array}{l}
\Phi_{1}^{0} \\
\Phi_{2}^{0}
\end{array}\right)=\frac{x^{2}}{4\left(a_{11} a_{22}-a_{12} a_{21}\right)}\left(\begin{array}{l}
-a_{22} \eta_{2}-a_{12} \eta_{1} \\
-a_{21} \eta_{2}-a_{11} \eta_{1}
\end{array}\right),
$$$$
\left(\begin{array}{c}
\Phi_{1}^{2 K_{1}} \\
\Phi_{2}^{2 K_{1}}
\end{array}\right)
$$

$$
=\frac{x^{2}}{4 \operatorname{det}\left(M_{2 K_{1}}\right)}\left(\begin{array}{l}
\left(\lambda_{0} \rho_{2 K_{1}}-a_{22}\right) \eta_{2}-a_{12} \eta_{1} \\
-a_{21} \eta_{2}+\left(\rho_{2 K_{1}}-a_{11}\right) \eta_{1}
\end{array}\right),
$$

$$
\left(\begin{array}{c}
\Phi_{1}^{2 K_{2}} \\
\Phi_{2}^{2 K_{2}}
\end{array}\right)
$$

$$
\begin{gathered}
=\frac{x^{2}}{4 \operatorname{det}\left(M_{2 K_{2}}\right)}\left(\begin{array}{c}
\left(\lambda_{0} \rho_{2 K_{2}}-a_{22}\right) \eta_{2}-a_{12} \eta_{1} \\
-a_{21} \eta_{2}+\left(\rho_{2 K_{2}}-a_{11}\right) \eta_{1}
\end{array}\right), \\
\left(\begin{array}{c}
\Phi_{1}^{2 K} \\
\Phi_{2}^{2 K}
\end{array}\right)=\frac{x^{2}}{4 \operatorname{det}\left(M_{2 K}\right)}\left(\begin{array}{c}
\left(\lambda_{0} \rho_{2 K}-a_{22}\right) \eta_{2}-a_{12} \eta_{1} \\
-a_{21} \eta_{2}+\left(\rho_{2 K}-a_{11}\right) \eta_{1}
\end{array}\right) .
\end{gathered}
$$

Inserting (66) into (59), by (60) and (61) we get

$$
\begin{aligned}
\left\langle G\left(x \varphi_{K}+\Phi\right), \varphi_{K}^{*}\right\rangle= & \frac{9 L_{1} L_{2}}{64} x^{3} \widetilde{H}\left(\xi, \xi^{*}\right) \\
& +\frac{L_{1} L_{2}}{16} x^{3} H\left(\xi, \xi^{*}\right)+o\left(x^{3}\right),
\end{aligned}
$$

where $\widetilde{H}\left(\xi, \xi^{*}\right)=a_{3} \xi_{1}^{*} \xi_{1} \xi_{2}{ }^{2}+a_{4} \eta_{1} \xi_{1}{ }^{2} \xi_{2}+a_{5} \xi_{1}^{*} \xi_{1}^{3}+b_{3} \eta_{2} \xi_{1}{ }^{2} \xi_{2}+$ $b_{4} \xi_{2}^{*} \xi_{1} \xi_{2}^{2}+b_{5} \xi_{2}^{*} \xi_{2}{ }^{3}$ and $H\left(\xi, \xi^{*}\right)$ is in Appendix.

In view of (70), it follows that

$$
\begin{aligned}
\left\langle\phi_{K}, \phi_{K}^{*}\right\rangle & =\left[a_{12} a_{21}+\left(a_{11}-\rho_{K}\right)^{2}\right] \int_{\Omega} e_{K}^{2} d x \\
& =\frac{L_{1} L_{2}}{4}\left[a_{12} a_{21}+\left(a_{11}-\rho_{K}\right)^{2}\right] .
\end{aligned}
$$

Hence, by (73) the reduced equation (13) is expressed as

$$
\frac{d x}{d t}=\beta_{1 K}(\lambda) x+\eta x^{3}+o\left(x^{3}\right) .
$$

Based on Theorem A.1 in [16], this theorem follows from (73). The proof is complete.

\section{Concluding Remarks and Example}

In this paper, we have studied the Turing bifurcation introduced by Alan Turing and attractor bifurcation developed by $\mathrm{Ma}$ and Wang [13] of gene propagation population model governed by reaction-diffusion equation.

Theorems 2 and 4 tell us that the critical value $\lambda_{0}$ of diffusion constant given by

$$
\lambda_{0}=\frac{a_{22} \rho_{K}-\left(a_{11} a_{22}-a_{12} a_{21}\right)}{\left(\rho_{K}-a_{11}\right) \rho_{K}}
$$




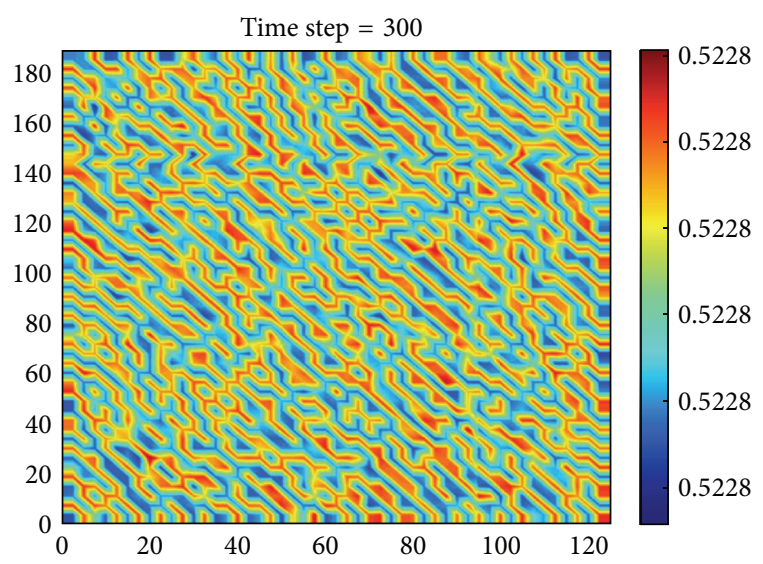

(a) $\lambda=13$

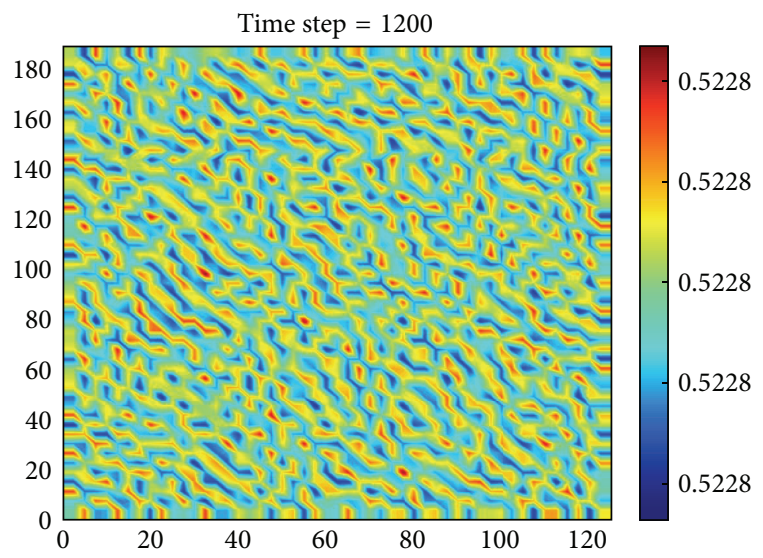

(b) $\lambda=15.86209390$

FIGURE 1: Turing patterns in two dimensions of system (9) with $\gamma_{i j}$ defined by (75). plays a crucial role in determining the attractor bifurcation and Turing bifurcation when the diffusion parameter $\lambda$ crosses the critical value $\lambda_{0}$, and the uniform stationary state $(a, b)$ loses its stability, which yields the Turing or attractor bifurcation.

We give some examples to illustrate our main theories.

Example 5. In system (9), we let

$$
\begin{aligned}
& \gamma_{11}=\frac{2773376069253}{499999999994}, \\
& \gamma_{22}=\frac{3789926287167}{499999999994}, \\
& \gamma_{33}=\frac{810843022017}{45454545454}, \\
& \gamma_{23}=\frac{4789523227559}{249999999997}, \\
& \gamma_{12}=\frac{6952222073553}{499999999994}, \\
& \gamma_{13}=\frac{436265576135}{249999999997},
\end{aligned}
$$

and then the steady state $(a, b)$ of system (9) is

$$
\begin{aligned}
& a=\frac{732070686076807741207077}{1417067211664436233221844}, \\
& b=\frac{380119934758603124485687}{708533605832218116610922},
\end{aligned}
$$

where $0<a$ and $b<1$.

If we set $\Omega=(0,40 \pi) \times(0,60 \pi)$, then $\rho_{K}=13 / 14400$, where $K=(1,1)$, and then

$$
\lambda_{0}=\frac{814910923617150223684573395768233809936382690140395479526347826117214934665200}{262055733351368170238516966160634367981740693955623134829331749178881678669} \approx 3109.685536
$$

By Theorem 1, we obtain that the transition conditions are satisfied.

From Theorem $4, \eta \approx-4527.181405<0$, and system (22) has a continuous transition from $\left(0, \lambda_{0}\right)$, which is an attractor bifurcation.

Since $\rho_{K}+a_{11}<0$, we infer that if $\lambda<\lambda_{0}$, then $\beta_{1 K}(\lambda)>0$, and the Turing instability occurs. By numerical simulation, we have shown that the gene population model is able to sustain Turing patterns (Figure 1).

\section{Appendix}

\section{The Expressions of $H\left(\xi, \xi^{*}\right)$}

The Expressions of $H\left(\xi, \xi^{*}\right)$ are as follows:

$$
\begin{aligned}
H\left(\xi, \xi^{*}\right)= & 4 a_{3} \xi_{1}^{*} b \Phi_{1}^{2 K_{1}} \xi_{2}+4 b \xi_{2}^{*} \Phi_{1}^{2 K_{1}} a_{5} \xi_{2} \\
& +4 \xi_{2}^{*} a_{5} b \xi_{1} \Phi_{2}^{2 K_{1}}+4 \xi_{2}^{*} a_{4} b \xi_{1} \Phi_{1}^{2 K_{2}}
\end{aligned}
$$

$$
\begin{aligned}
& +4 \xi_{2}^{*} a_{4} b \Phi_{1}^{2 K_{2}} \xi_{2}+4 \xi_{2}^{*} a_{4} b \xi_{1} \Phi_{2}^{2 K_{2}} \\
& +2 \xi_{2}^{*} a_{5} b \xi_{2} \Phi_{2}^{2 K}+2 \xi_{1}^{*} a_{4} b \Phi_{1}^{2 K} \xi_{2} \\
& +4 \xi_{1}^{*} a_{3} a \xi_{2} \Phi_{2}^{2 K_{2}}+4 \xi_{1}^{*} a_{3} b \xi_{2} \Phi_{2}^{2 K_{1}} \\
& +4 \xi_{2}^{*} a_{5} b \xi_{1} \Phi_{2}^{2 K_{2}}+6 \xi_{1}^{*} a_{5} b \xi_{1} \Phi_{1}^{2 K} \\
& +2 \xi_{2}^{*} a_{4} a \Phi_{1}^{2 K} \xi_{2}+4 \xi_{1}^{*} a_{4} b \xi_{1} \Phi_{1}^{2 K_{1}} \\
& +12 \xi_{1}^{*} a_{5} b \xi_{1} \Phi_{1}^{2 K_{1}}+2 \xi_{1}^{*} a_{3} b \xi_{2} \Phi_{2}^{2 K} \\
& +2 \xi_{1}^{*} a_{4} b \xi_{1} \Phi_{1}^{2 K}+24 \xi_{1}^{*} a_{5} b \xi_{1} \Phi_{1}^{0} \\
& +2 \xi_{1}^{*} a_{3} b \xi_{1} \Phi_{2}^{2 K}+12 a_{3} b \xi_{2}^{*} \xi_{2} \Phi_{2}^{2 K_{1}} \\
& +24 a_{3} b \Phi_{2}^{0} \xi_{2}^{*} \xi_{2}+2 \xi_{2}^{*} b_{2} \xi_{1} \Phi_{2}^{2 K_{2}} \\
& +4 \xi_{2}^{*} a_{4} b \Phi_{1}^{2 K_{1}} \xi_{2}+4 \xi_{1}^{*} a_{4} b \xi_{1} \Phi_{2}^{2 K_{1}} \\
& +12 \xi_{1}^{*} a_{5} b \xi_{1} \Phi_{1}^{2 K_{2}}+4 \xi_{1}^{*} a_{4} b \Phi_{1}^{2 K_{2}} \xi_{2}
\end{aligned}
$$




$$
\begin{aligned}
& +8 \xi_{1}^{*} a_{4} b \xi_{1} \Phi_{1}^{0}+8 \xi_{2}^{*} a_{5} b \xi_{1} \Phi_{2}^{0} \\
& +2 \xi_{2}^{*} a_{4} b \xi_{1} \Phi_{1}^{2 K}+8 \xi_{2}^{*} a_{4} b \xi_{1} \Phi_{2}^{0} \\
& +4 \xi_{1}^{*} a_{4} b \xi_{1} \Phi_{2}^{2 K_{2}}+4 a_{3} \Phi_{1}^{2 K_{2}} \xi_{1}^{*} b \xi_{2} \\
& +12 a_{3} b \xi_{2}^{*} \xi_{2} \Phi_{2}^{2 K_{2}}+4 \xi_{2}^{*} a_{4} b \xi_{1} \Phi_{2}^{2 K_{1}} \\
& +4 \xi_{1}^{*} a_{3} b \xi_{1} \Phi_{2}^{2 K_{1}}+4 \xi_{1}^{*} a_{4} b \Phi_{1}^{2 K_{1}} \xi_{2} \\
& +4 \Phi_{1}^{2 K_{2}} b \xi_{2}^{*} a_{5} \xi_{2}+4 \xi_{2}^{*} a_{5} b \xi_{2} \Phi_{2}^{2 K_{2}} \\
& +4 \xi_{1}^{*} a_{4} b \xi_{1} \Phi_{1}^{2 K_{2}}+6 \xi_{2}^{*} a_{3} b \xi_{2} \Phi_{2}^{2 K} \\
& +4 \xi_{1}^{*} a_{3} b \xi_{1} \Phi_{2}^{2 K_{2}}+4 \xi_{2}^{*} a_{5} b \xi_{2} \Phi_{2}^{2 K_{1}} \\
& +2 \Phi_{1}^{2 K} b \xi_{2}^{*} a_{5} \xi_{2}+4 \xi_{2}^{*} b_{2} \Phi_{1}^{0} \xi_{2} \\
& +8 \xi_{1}^{*} a_{1} \xi_{1} \Phi_{1}^{0}+2 \xi_{1}^{*} a_{2} \xi_{1} \Phi_{2}^{2 K_{2}} \\
& +2 \xi_{1}^{*} a_{2} \Phi_{1}^{2 K_{2}} \xi_{2}+2 b_{2} \Phi_{1}^{2 K_{2}} \xi_{2}^{*} \xi_{2} \\
& +4 \xi_{2}^{*} b_{2} \xi_{1} \Phi_{2}^{0}+2 \xi_{1}^{*} a_{2} \xi_{1} \Phi_{2}^{2 K_{1}} \\
& +4 \xi_{2}^{*} b_{1} \xi_{2} \Phi_{2}^{2 K_{2}}+4 \xi_{1}^{*} a_{2} \Phi_{1}^{0} \xi_{2} \\
& +4 \xi_{1}^{*} a_{1} \xi_{1} \Phi_{1}^{2 K_{1}}+2 \xi_{1}^{*} a_{2} \Phi_{1}^{2 K_{1}} \xi_{2} \\
& +\Phi_{1}^{2 K} b_{2} \xi_{2}^{*} \xi_{2}+4 \xi_{1}^{*} a_{1} \xi_{1} \Phi_{1}^{2 K_{2}} \\
& +\xi_{2}^{*} b_{2} \xi_{1} \Phi_{2}^{2 K}+2 \xi_{2}^{*} b_{1} \xi_{2} \Phi_{2}^{2 K} \\
& +4 \xi_{1}^{*} a_{2} \xi_{1} \Phi_{2}^{0}+\xi_{1}^{*} a_{2} \Phi_{1}^{2 K} \xi_{2} \\
& +2 b_{2} \xi_{2}^{*} \Phi_{1}^{2 K_{1}} \xi_{2}+2 \xi_{1}^{*} a_{1} \xi_{1} \Phi_{1}^{2 K} \\
& +8 \Phi_{2}^{0} \xi_{2}^{*} b_{1} \xi_{2}+2 \xi_{2}^{*} b_{2} \xi_{1} \Phi_{2}^{2 K_{1}} \\
& +4 \xi_{2}^{*} b_{1} \xi_{2} \Phi_{2}^{2 K_{1}}+8 \xi_{1}^{*} a_{3} b \xi_{2} \Phi_{2}^{0} \\
& +2 \xi_{1}^{*} a_{4} b \xi_{1} \Phi_{2}^{2 K}+2 \xi_{2}^{*} a_{5} b \xi_{1} \Phi_{2}^{2 K} \\
& +2 \xi_{2}^{*} a_{4} b \xi_{1} \Phi_{2}^{2 K}+2 \Phi_{1}^{2 K} a_{3} \xi_{1}^{*} b \xi_{2} \\
& +\xi_{1}^{*} a_{2} \xi_{1} \Phi_{2}^{2 K}+8 \xi_{1}^{*} a_{3} b \Phi_{1}^{0} \xi_{2} \\
& +8 \xi_{1}^{*} a_{3} b \xi_{1} \Phi_{2}^{0}+8 \xi_{1}^{*} a_{4} b \xi_{1} \Phi_{2}^{0} \\
& +8 \xi_{2}^{*} a_{4} b \xi_{1} \Phi_{1}^{0}+8 \xi_{1}^{*} a_{4} b \Phi_{1}^{0} \xi_{2} \\
& +8 \xi_{2}^{*} a_{5} b \Phi_{1}^{0} \xi_{2}+8 \xi_{2}^{*} a_{4} b \Phi_{1}^{0} \xi_{2} \\
& +8 \xi_{2}^{*} a_{5} b \xi_{2} \Phi_{2}^{0}+4 \xi_{2}^{*} a_{4} b \xi_{1} \Phi_{1}^{2 K_{1}} .
\end{aligned}
$$

\section{Competing Interests}

The author declares that there is no conflict of interests regarding the publication of this paper.

\section{Acknowledgments}

This paper was partially supported by National Natural Science Foundation of China (Grants no. 11401062 and no. 11371386), Research Fund for the National Natural Science Foundation of Chongqing CSTC (Grant no. cstc2014jcyjA0080), Scientific and Technological Research Program of Chongqing Municipal Education Commission (Grant no. KJ1400937), and the Scientific Research Foundation of CQUT (Grant no. 2012ZD37).

\section{References}

[1] R. A. Fisher, "The wave of advance of advantageous genes," Annals of Eugenics, vol. 7, no. 4, pp. 355-369, 1937.

[2] A. D. Bazykin, "Hypothetical mechanism of speciaton," Evolution, vol. 23, no. 4, pp. 685-687, 1969.

[3] J. Piálek and N. H. Barton, “The spread of an advantageous allele across a barrier: the effects of random drift and selection against heterozygotes," Genetics, vol. 145, no. 2, pp. 493-504, 1997.

[4] T. Nagylaki, "Conditions for the existence of clines," Genetics, vol. 80, no. 3, pp. 595-615, 1975.

[5] T. Nagylaki and J. F. Crow, "Continuous selective models," Theoretical Population Biology, vol. 5, no. 2, pp. 257-283, 1974.

[6] B. H. Bradshaw-Hajek and P. Broadbridge, "A robust cubic reaction-diffusion system for gene propagation," Mathematical and Computer Modelling, vol. 39, no. 9-10, pp. 1151-1163, 2004.

[7] B. H. Bradshaw-Hajek, P. Broadbridge, and G. H. Williams, "Evolving gene frequencies in a population with three possible alleles at a locus," Mathematical and Computer Modelling, vol. 47, no. 1-2, pp. 210-217, 2008.

[8] P. Broadbridge, B. H. Bradshaw, G. R. Fulford, and G. K. Aldis, "Huxley and Fisher equations for gene propagation: an exact solution," The Anziam Journal, vol. 44, no. 1, pp. 11-20, 2002.

[9] A. Kolmogorov, I. Petrovsky, and N. Piscounov, "Études de l'equation aved croissance de la quantité de matière et son application à un problème biologique," Moscow University Mathematics Bulletin, vol. 1, pp. 1-25, 1937.

[10] M. Slatkin, "Gene flow and selection in a cline," Genetics, vol. 75, no. 4, pp. 733-756, 1973.

[11] J. M. Smith, Mathematical Ideas in Biology, Cambridge University Press, Cambridge, UK, 1968.

[12] R. A. Littler, "Loss of variability at one locus in a finite population," Mathematical Biosciences, vol. 25, no. 1-2, pp. 151$163,1975$.

[13] T. Ma and S. Wang, Bifurcation Theory and Applications, vol. 53 of Nonlinear Science Series A, World Scientific, Beijing, China, 2005.

[14] T. Ma and S. Wang, Stability and Bifurcation of the Nonlinear Evolution Equations, Science Press, Singapore, 2007 (Chinese).

[15] A. Turing, "The chemical basis of morphogenesis," Philosophical Transactions of the Royal Society of London B, vol. 237, no. 641, pp. 37-52, 1952.

[16] T. Ma and S. Wang, "Dynamic phase transition theory in PVT systems," Indiana University Mathematics Journal, vol. 57, no. 6, pp. 2861-2889, 2008. 


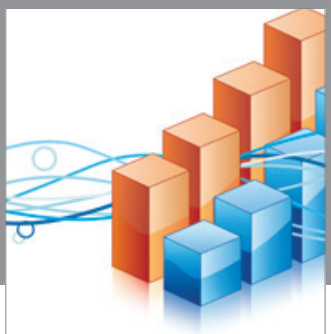

Advances in

Operations Research

vatem alat4

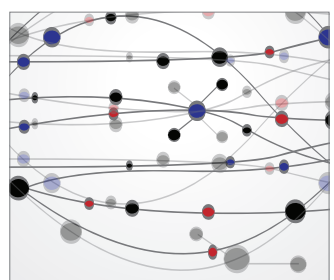

\section{The Scientific} World Journal
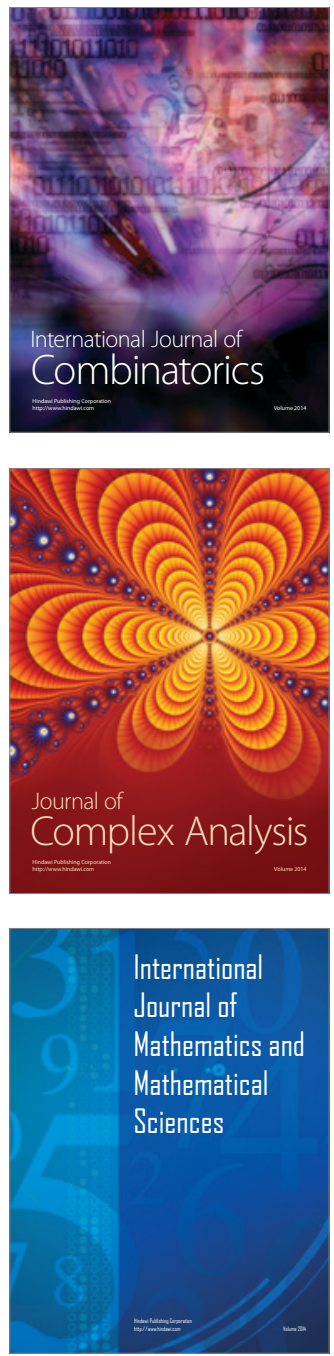
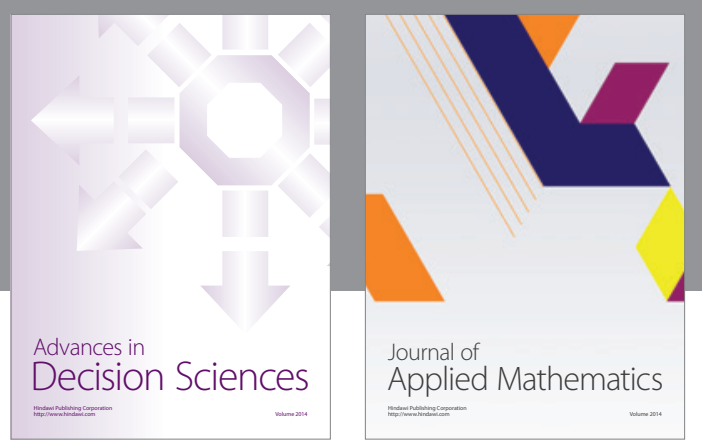

Algebra

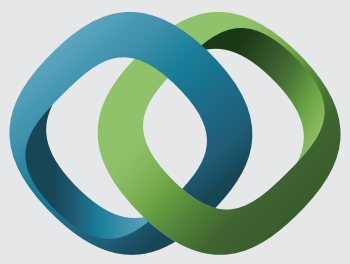

\section{Hindawi}

Submit your manuscripts at

http://www.hindawi.com
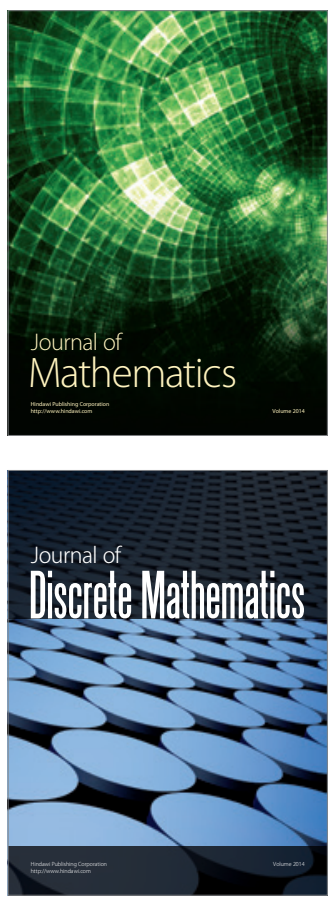

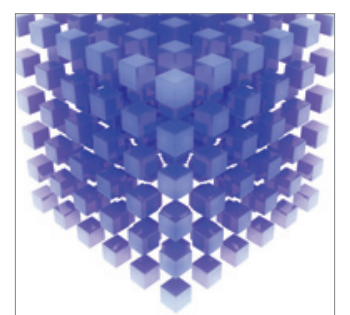

Mathematical Problems in Engineering
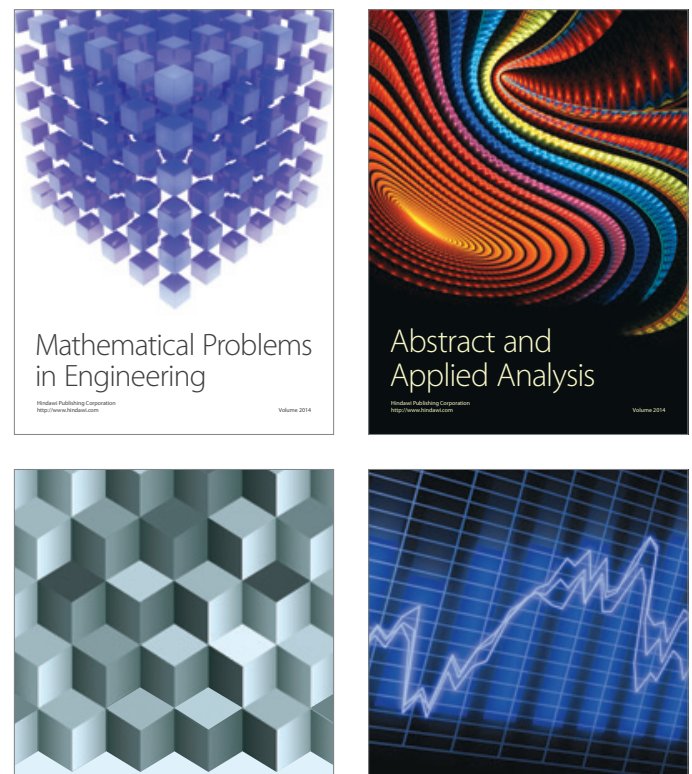

Journal of

Function Spaces

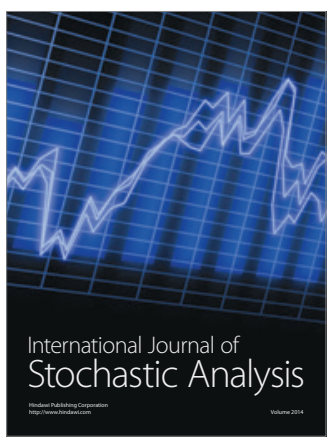

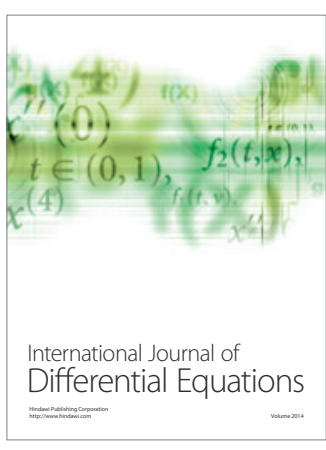
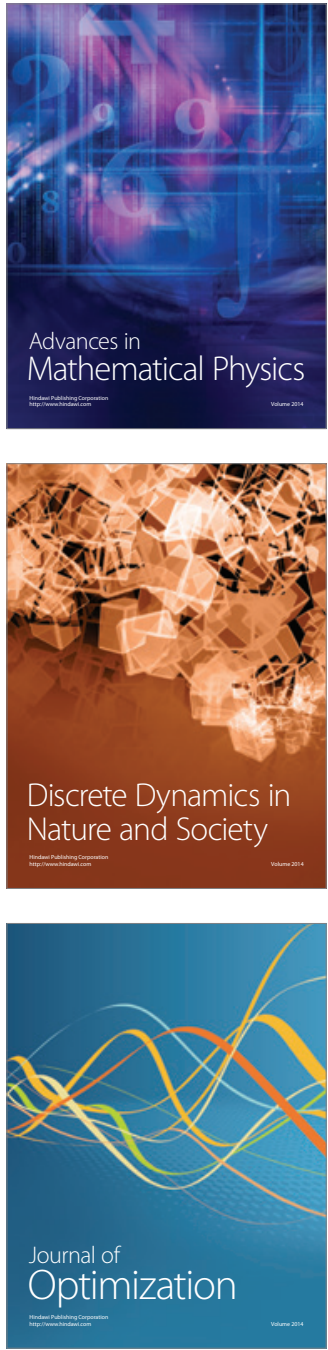